\title{
Infecções oportunistas por leveduras e perfil enzimático dos agentes etiológicos
}

\author{
Opportunistic yeast infections and enzymatic profile \\ of the etiological agents
}

\author{
Danielle Patrícia Cerqueira Macêdo ${ }^{1}$, Aline Mary de Almeida Farias ${ }^{1}$, \\ Reginaldo Gonçalves de Lima Neto ${ }^{1}$, Vanessa Karina Alves da Silva, \\ André Ferraz Goiana Leal ${ }^{1}$ e Rejane Pereira Neves ${ }^{1}$
}

\begin{abstract}
RESUMO
Infecções por leveduras são freqüentes em imunocomprometidos, contudo espécies emergentes têm alterado o perfil epidemiológico. A habilidade de secretar proteases tem sido associada à patogenicidade do gênero Candida. Esta pesquisa teve como objetivos diagnosticar leveduroses em pacientes imunocomprometidos e avaliar a virulência dos agentes etiológicos baseado em teste de secreção de protease utilizando soro de albumina bovina como substrato. Do total de 104 pacientes estudados, 19 apresentaram episódios de leveduroses. 0 trato respiratório (63,2\%), seguido pelo trato urinário (10,5\%) foram os locais mais comuns de infecção. Candida albicans, Candida parapsilosis, Candida tropicalis e espécies emergentes como Candida krusei e Candida guilliermondii foram isoladas. Cinco isolados de Candida parapsilosis e um de Candida albicans e Candida guilliermondii exibiram alta atividade enzimática. Concluímos que a caracterização enzimática de isolados de Candida pode ser um útil marcador prognóstico, especialmente em imunocomprometidos, uma vez que leveduroses nestes pacientes são geralmente graves.
\end{abstract}

Palavras-chaves: Infecções por leveduras. Imunocomprometidos. Atividade proteásica. Patogenicidade.

\begin{abstract}
Yeast infections are common in immunocompromised patients, although emerging species have been changing the epidemiological profile. The ability to secrete proteinases has been associated with pathogenicity within the genus Candida. This study had the aims of diagnosing yeast infections in immunocompromised patients and evaluating the virulence of the etiological agents, based on a proteinase secretion assay using bovine serum albumin as a substrate. Out of a total of 104 patients studied, 19 presented episodes of yeast infection. The respiratory tract (63.2\%), followed by the urinary tract (10.5\%), were the most common sites of infection. Candida albicans, Candida parapsilosis, Candida tropicalis and emerging species such as Candida krusei and Candida guilliermondii were isolated. Five isolates of Candida parapsilosis and one of Candida albicans and Candida guilliermondii exhibited high enzymatic activity. We conclude that enzymatic characterization of Candida isolates may be a useful prognostic marker, especially among immunocompromised individuals, since yeast infections in such patients are generally serious.
\end{abstract}

Key-words: Yeast infections. Immunocompromised patients. Proteinase activity. Pathogenicity.

A incidência de infecções oportunistas por leveduras tem se tornado crescente, devido à maior agressividade no tratamento de neoplasias, transplante de órgãos, síndrome da imunodeficiência adquirida (SIDA), lupus eritematoso sistêmico (LES), entre outras doenças $^{2125}$.

Infecções sistêmicas causadas por leveduras do gênero Candida são freqüentes no hospedeiro imunocomprometido, com aproximadamente 20 espécies reconhecidamente patogênicas destacando-se Candida albicans, Candida tropicalis e Candida

1. Departamento de Micologia, Centro de Ciências Biológicas, Universidade Federal de Pernambuco, Recife, PE.

Endereço para correspondência: Dra Rejane Pereira Neves. Rua José Paraíso 135/01, Boa Viagem, 51030-390 Recife, PE.

Fax: $55812126-8482$

e-mail: rejadel@yahoo.com.br

Recebido para publicação em 05/12/2008

Aceito em 20/03/2009 glabrata $^{82124}$, sendo consideradas de difícil diagnóstico com altas taxas de morbidade e mortalidade, apesar das terapias antifúngicas $^{29}$.

Atualmente, podemos evidenciar uma mudança marcante no perfil epidemiológico das leveduroses ao serem relatados casos de infecções por espécies emergentes ${ }^{621} 29$.

Estas leveduras apresentam a habilidade de passar da condição de comensal a patógena quando sob condições favoráveis no hospedeiro, dependendo para isso dos diversos fatores de virulência, incluindo a secreção de enzimas hidrolíticas como proteases ${ }^{15}$.

Atividade proteásica relaciona-se diretamente com a degradação de componentes teciduais como colágeno, queratina e mucina, além de componentes imunológicos como anticorpos, complemento e citocinas. A habilidade de leveduras de interesse médico em secretar proteases tem sido associada a fatores de patogenicidade $^{30}$. 
O presente estudo objetivou diagnosticar infecções oportunistas por leveduras e avaliar a virulência dos agentes etiológicos quanto à produção de protease.

\section{MATERIAL E MÉTODOS}

Pacientes. Foram avaliados pacientes imunocomprometidos internados em diversos setores do Hospital das Clínicas de Pernambuco (HCPE), no período de Janeiro de 2006 a janeiro de 2007, com sintomatologia e/ou lesões sugestivas para leveduroses. Os quadros clínicos mais comuns foram definidos de acordo com o local de acometimento: infecções do trato respiratório, trato urinário, trato gastrointestinal e casos de lesões localizadas ou disseminação.

A coleta das amostras clínicas foi realizada de acordo com o local e características das lesões, após aprovação pelo Comitê de Ética em Saúde da Universidade Federal de Pernambuco (UFPE). No Laboratório de Micologia Médica da UFPE, as amostras foram processadas através de método padrão (exame direto e cultura) para diagnóstico micológico segundo Lacaze cols ${ }^{19}$. 0 exame direto das amostras obtidas foi realizado a partir de preparações em lâminas a fresco, clarificadas com solução aquosa de hidróxido de potássio (KOH) a 20\% ou contrastadas com tinta Nankin. Posteriormente, o material para análise foi semeado em quadriplicata nos meios ágar Sabouraud (Difco) e Brain Heart Infusion (Difco), ambos contendo 50mg/L de cloranfenicol. As placas foram mantidas a $30^{\circ} \mathrm{C}$ e $37^{\circ} \mathrm{C}$ durante dez dias.

Isolados. A identificação dos agentes etiológicos foi realizada segundo Barnett e cols3 e Hoog e cols ${ }^{14}$ através da observação das características macroscópicas (aspecto, coloração e bordos das colônias), microscópicas (presença de pseudomicélio, micélio verdadeiro e produção de clamidosporos) e fisiológicas (assimilação de fontes de nitrogênio e carbono, fermentação de fontes de carbono e produção de urease). As leveduras também foram avaliadas através de método automatizado VITEK 120 (BioMérieux).

Detecção de protease. Testes preliminares de patogenicidade foram desenvolvidos através da detecção da enzima protease utilizando-se meio indutor contendo soro de albumina bovina (BSA, fração V, Sigma, St Louis, MO) como substrato ${ }^{1}$. As leveduras isoladas foram suspensas em 1,0mL de água destilada esterilizada e contadas em câmara de Neubauer obtendo-se concentração de 106 células/ mL. Posteriormente, foram inoculadas em poços no referido meio de cultura e incubadas a temperatura de $37^{\circ} \mathrm{C}$, com o objetivo de detectar a formação de um halo transparente. 0 teste foi realizado em triplicata com leitura ao final de sete dias e cálculo da zona de atividade (ZA) que equivale à relação entre o diâmetro da colônia e o diâmetro da zona de precipitação. Os valores da ZA foram agrupados em quatro classes: ausente $(-; 1,0)$; muito baixo $(+; 0,90-0,99)$; baixo $(++$; $0,80-0,89)$; alto $(+++; 0,70-0,79)^{28}$.

\section{RESULTADOS}

Foram avaliados 104 pacientes internados no HCPE os quais apresentaram diversas doenças de base e fizeram uso prolongado de drogas que conduziram a estados de imunossupressão permanente ou transitória, no período de janeiro de 2006 a janeiro de 2007. Destes, 19 pacientes exibiram estado de neutropenia e apresentaram na microscopia direta das amostras clínicas numerosas células de leveduras brotantes, com formação de pseudomicélio e micélio verdadeiro. Foi obtido um isolado de levedura para cada caso avaliado, com exceção de um paciente que apresentou lesões em sítios corpóreos distintos (Tabela 1). A idade variou entre 16 e 86 anos, destacando-se o sexo feminino com $53 \%$ dos casos.

TABELA 1

\begin{tabular}{|c|c|c|c|c|c|c|}
\hline Paciente & Sexo & Idade & Doença de base & Local & Espécies de Candida & $\mathbf{Z A}$ \\
\hline 1 & $\mathrm{M}$ & 32 & insuficiência renal & TGI & Candida albicans & ++ \\
\hline 2 & M & 33 & tuberculose pulmonar & TR & Candida kruse $i$ & ++ \\
\hline 3 & M & 72 & câncer e diabetes mellitus & TR & Candida parapsilosis & +++ \\
\hline 4 & $\mathrm{~F}$ & 55 & hipotireoidismo & $\mathrm{TU}$ & Candida albicans & ++ \\
\hline 5 & M & 26 & AIDS & TR & Candida tropicalis & + \\
\hline 6 & $\mathrm{~F}$ & 50 & lupus eritematoso & disseminada & Candida albicans & ++ \\
\hline 7 & M & 24 & tuberculose pulmonar & TR & Candida albicans & ++ \\
\hline 8 & M & 58 & câncer & Pele & Candida guilliermondii & +++ \\
\hline 9 & $\mathrm{~F}$ & 60 & diabetes mellitus & TR & Candida parapsilosis & +++ \\
\hline 10 & $\mathrm{~F}$ & 16 & lupus eritematoso & TR & Candida parapsilosis & + \\
\hline 11 & M & 26 & AIDS & TR & Candida albicans & +++ \\
\hline 12 & M & 86 & osteomielite & tecido ósseo e pele & Candida parapsilosis & +++ \\
\hline 13 & $\mathrm{~F}$ & 35 & tuberculose pulmonar & TR & Candida albicans & ++ \\
\hline 14 & $\mathrm{~F}$ & 63 & diabetes mellitus & $\mathrm{TU}$ & Candida albicans & +++ \\
\hline 15 & $\mathrm{~F}$ & 27 & insuficiência renal & TR & Candida tropicalis & + \\
\hline 16 & $\mathrm{~F}$ & 52 & diabetes mellitus & disseminada & Candida parapsilosis & +++ \\
\hline 17 & M & 31 & pneumonia & TR & Candida albicans & ++ \\
\hline 18 & $\mathrm{~F}$ & 82 & insuficiência renal & TR & Candida albicans & ++ \\
\hline 19 & $\mathrm{~F}$ & 20 & AIDS & TR & Candida albicans & - \\
\hline
\end{tabular}

ZA: zona de atividade proteásica, F: sexo feminino, M: sexo masculino, TGI: trato gastrointestinal, TR: trato respiratório, TU: trato urinário. AIDS: síndrome da imunodeficiência adquirida, -: ausente, +: muito baixa, ++: baixa, +++: alta. 
As condições de imunossupressão relacionadas às leveduroses diagnosticadas incluíram insuficiência renal crônica e aguda, tuberculose pulmonar, SIDA, diabetes mellitus, LES, entre outras doenças de base, associadas aos seus respectivos tratamentos. Quanto à localização das leveduroses, verificamos que o local mais acometido foi o trato respiratório com $63,2 \%$, seguido pelo trato urinário com $10,5 \%$ dos casos.

Os isolados de leveduras obtidos foram identificados como Candida albicans (50\%), Candida parapsilosis (30\%) e Candida tropicalis (10\%). Outras espécies como Candida krusei (5\%) e Candida guilliermondii (5\%), consideradas patógenos emergentes, também foram isoladas.

Do total de 20 isolados de Candida testados, 100\% foram produtores de protease, variando os valores de ZA entre as espécies testadas e o local da infecção, destacando-se cinco isolados de Candida parapsilosis e um de Candida albicans e Candida guilliermondii que exibiram alta atividade desta enzima (Tabela 1).

Os valores de ZA destes isolados demonstraram relação direta com o grau de agressividade das leveduroses. Cinco dos seis isolados de Candida parapsilosis apresentaram alta atividade proteásica, dos quais três foram obtidos do trato respiratório (secreção traqueal e lavado brônquico) e dois de tecido ósseo e pele. De forma semelhante, Candida albicans obtida de urina de paciente com diabetes mellitus e Candida guilliermondii, obtida através de biópsia da pele de paciente com câncer fazendo uso de quimioterápicos, também apresentaram valores elevados de protease.

\section{DISCUSSÃO}

As leveduras do gênero Candida equivalem a 8-10\% da causa das infecções oportunistas e permanecem como importantes patógenos nosocomiais ${ }^{5}{ }^{16}$. Alta incidência de infecções oportunistas por leveduras tem sido evidenciada sob manifestações clínicas e fatores de risco relacionados à neutropenia como diabetes mellitus, tuberculose pulmonar, LES, AIDS, uso de drogas imunossupressoras, mucosite induzida por quimioterapia ou altas doses de irradiação ${ }^{21727}$.

Vários aspectos da imunidade são alterados em pacientes com diabetes e tuberculose. Nestas doenças, a função de leucócitos polimorfonucleares é deprimida, ocorrendo nos casos de diabetes quando a acidose está presente. A aderência leucocitária, a quimiotaxia e a fagocitose podem ser afetadas conduzindo a infecções oportunistas por leveduras ${ }^{720}$.

Estudos prévios desenvolvidoscompacientesimunocomprometidos de ambos os sexos e com infecções fúngicas do trato respiratório revelaram predominância no sexo feminino ${ }^{12} 16$, corroborando com os casos avaliados nesta pesquisa.

Estruturas dos agentes etiológicos evidenciadas ao exame direto, como pseudomicélio e micélio verdadeiro, reforçam a condição de infecção dos pacientes, afastando a possibilidade de colonização. Gow ${ }^{11}$ e Lacaz $^{19}$ consideram que estas diferenciações facilitam a adesão, invasão e disseminação no tecido hospedeiro.
Em concordância com os resultados de Hamza e cols ${ }^{13}$ Candida albicans foi a espécie mais frequentemente isolada a partir de infecções do trato respiratório, seguida por Candida glabrata e Candida krusei, demonstrando que a emergência de espécies não-albicans tem produzido efeito relevante nas condutas clínicas ${ }^{9}$. Estudo comparativo de infecções disseminadas em pacientes imunocomprometidos verificou que a taxa de mortalidade associada com Candida krusei foi de 49\%, se comparada à taxa de apenas $28 \%$ por Candida albicans ${ }^{8}$.

Dentre os atributos associados à virulência do gênero Candida, destaca-se a elaboração de enzimas proteolíticas e estudos experimentais têm sugerido que elevada produção de proteases é um importante aspecto de Candida para interação com tecido hospedeiro e virulência ${ }^{4} 1022$.

Nutrientes contidos no BSA resultam em níveis elevados de protease, demonstrando o potencial de invasão dos agentes etiológicos ${ }^{22}$. Zaugg e cols ${ }^{31}$ avaliaram atividade desta enzima por espécies não-albicans, não sendo observadas variações. Diferenças ou ausência na expressão desta enzima por leveduras isoladas de pacientes HIV-positivos podem ser explicadas pelo uso extensivo de inibidores de proteases ${ }^{32}$.

Pesquisa recente desenvolvida por Nawrot e $\operatorname{cols}^{23}$ com pacientes imunocomprometidos demonstrou que leveduras isoladas de sangue produziram significativamente menos protease que as obtidas de infecções do trato respiratório, corroborando com nossos resultados. Todavia, Oksuz e cols ${ }^{26}$ verificaram que apenas Candida albicans isolada do trato urogenital e pele de imunocompetentes apresentam alta expressão proteásica.

De acordo com Silva e cols ${ }^{30}$, esta metodologia pode ser utilizada na caracterização fenotípica de leveduras e, portanto, servir como um marcador epidemiológico de infecções por Candida, especialmente em ambiente hospitalar que predispõe os imunocomprometidos a leveduroses graves e de repetição devido aos procedimentos invasivos a que estes pacientes estão $\operatorname{expostos}^{18}$.

O isolamento de espécies de Candida de amostras clínicas de pacientes com reduzida resistência imunológica deve ser considerado um achado significante, tendo correspondido na maioria dos casos a infecções secundárias ${ }^{27}$.

\section{REFERÊNCIAS}

1. Aoki S, Ito-Kuwa S, Nakamura K, Ninomiya K, Vidotto V. Extracellular proteolytic activity of Cryptococcus neoformans. Mycophatologia 128: 143-150, 1994.

2. Barnes PD, Marr KA. Risk, diagnosis and outcomes of invasive fungal infections in haematopoietic stem cell transplant recipients. British Journal of Haematology 139: 519-531, 2007.

3. Barnett JA, Paine RW, Yarrow D. Yeasts: Characteristics and Identification. Cambridge, Cambridge University Press, 2000.

4. Bramono K, Yamazaki M, Tsuboi R, Ogawa H. Comparison of proteinase, lipase and alpha-glucosidase activities from the clinical isolates of Candida species. Japanese Journal of Infectious Diseases 59: 73-76, 2006.

5. Cardona-Castro N, Revankar SG, Ortiz P, Cuervo C, Kirkpatrick WR, McAtee RK, Patterson TF. Proteinase detection, DNA typing and antimycotic susceptibility of Candida isolates from Colombian women with vulvovaginal candidiasis. Revista Iberoamericana de Micologia 19: 89-94, 2002. 
6. Carvalho AMR, Melo LRB, Moraes VL, Neves RP. Invasive Trichosporon cutaneum infection in an infant with wilms. Brazilian Journal of Microbiology 39: 1-2, 2008.

7. Chadeganipour M, Shadzi S, Dehghan P, Bijary J. The incidence of opportunistic fungi in patients suspected of tuberculosis. Mycoses 43: 269-272, 2000.

8. Colombo AL, Guimaraes T. Epidemiologia das infecções hematogênicas por Candida spp. Revista da Sociedade Brasileira de Medicina Tropical 36: 599-607, 2003.

9. Costa KRC, Ferreira JC, Komesu MC, Candido RC. Candida albicans and Candida tropicalis in oral candidosis: quantitative analysis, exoenzyme activity, and antifungal drug sensitivity. Mycopathologia 167: 73-79, 2009.

10. De Bernardis F, Sullivan PA, Cassone A. Aspartyl proteinases of Candida albicans and their role in pathogenicity. Medical Mycology 39: 303-313, 2001.

11. Gow NA. Candida albicans switches mates. Molecular Cell 10: 217-218, 2002

12. Gyanchandani A, Khan ZK, Prasad R. Fungal infections in immunocompromised patients with pulmonary tuberculosis as a secondary disease. Journal de Mycologie Medicale 8: 147-152, 1998.

13. Hamza OJM, Matee MIN, Moshi MJ, Simon ENM, Mugusi F, Mikx FHM, Helderman WHP, Rijs AJMM, Ven AJAM, Verweij PE. Species distribution and in vitro antifungal susceptibility of oral yeast isolates from Tanzanian HIV-infected patients with primary and recurrent oropharyngeal candidiasis. BMC Microbiology 8: 135-144, 2008.

14. Hoog GS, Guarro J, Gené J, Figueras MJ. Atlas of clinical fungi. Centraalbureau voor Schimmelculures/Universitat Rovira i Virgili, Utrecht/Reus, The Netherlands, 2000.

15. Kantarcioglu AS, Yucel A. Phospholipase and protease activities in clinical Candida isolates with reference to the source of strains. Mycoses 45: 160-165, 2002.

16. Khosravi AR, Yarahmadi S, Baiat M, Shokri H, Pourkabireh M. Factors affecting the prevalence of yeasts in the oral cavity of patients with diabetes mellitus. Journal de Mycologie Médicale 18: 83-88, 2008.

17. Koh AY, Kohler JR, Coogshall KT, Rooijen NV, Pier GB. Mucosal damage and neutropenia are required for Candida albicans dissemination. Plos pathogens 4: e35, 2008.

18. Kumar CPG, Kumar SSJ, Menon T. Phospholipase and proteinase activities of clinical isolates of Candida from immunocompromised patients. Mycopathologia 161: 213-218, 2006.
19. Lacaz CS, Porto E. Tratado de Micologia Médica. Editora Sarvier, São Paulo, 2002.

20. Levidiotou-Stefanou S. Fungal infections in patients with diabetes mellitus and renal diseases. Acta Microbiologica Hellenica 53: 83-88, 2008.

21. Macêdo DPC, Silva VKA, Farias AMA, Melo LRB, Wilheim AB, Neves RP. Candida glabrata esophagitis: new case reports and management. Brazilian Journal of Microbiology 39: 1-7, 2008.

22. Naglik J, Albrecht A, Bader O, Hube B. Candida albicans proteinases and hostpathogen interactions. Cell Microbiology 6: 915-926, 2004.

23. Nawrot U, SkaBa J, WBodarczyk K, Fonteyne PA, Nolard N, Nowicka J. Proteolytic activity of clinical Candida albicans isolates in relation to genotype and strain source. Polish Journal of Microbiology 57: 27-33, 2008.

24. Neves RP, Cavalcanti MAQ, Chaves GM, Magalhães OMC. Yeasts isolated from clinical samples of AIDS patients. Brazilian Journal of Microbiology 33: 363-364, 2002.

25. Nucci M, Marr KA. Emerging fungal diseases. Clinical Infectious Diseases 41: 521-526, 2005.

26. Oksuz S, Sahin I, Yildirim M, Gulcan A, Yavuz T, Kaya D, Koc AN. Phospholipase and Proteinase Activities in Different Candida Species Isolated from Anatomically Distinct Sites of Healthy Adults. Japanese Journal of Infectious Diseases 60:280$283,2007$.

27. Pappas PG. Invasive candidiasis. Infectious Disease Clinics of North America 20: 485-506, 2006

28. Price MF, Walkison ID, Gentry LO. Plate method for detection of phospholipase activity in Candida albicans. Sabouraudia 20: 7-14, 1982.

29. Richardson M, Lass-Florl C. Changing epidemiology of systemic fungal infections. Clinical Microbiology and Infection 14: 5-24, 2008.

30. Silva JO, Ferreira JC, Candido RC. Atividade enzimática, produção de slime e sensibilidade a antifúngicos de Candida sp. Revista da Sociedade Brasileira de Medicina Tropical 40: 354-355, 2007.

31. Zaugg C, Zepelin MB, Reichard U, Sanglard D, Monod M. Secreted aspartic proteinase family of Candida tropicalis. Infection and Immunity 69: 405-412, 2001.

32. Zepelin MB, Meyer I, Thomssen R, Würzner R, Sanglard D, Telenti A, Monod M. HIV-protease inhibitors reduce cell adherence of Candida albicans by inhibition of yeast secreted aspartic proteases. Journal of Investigative Dermatology 113: 747-751, 1999. 